\title{
Quantification of stimuli
}

Ash MM. Quantification of stimuli. Endod Dent Traumatol 1986; 2: 153-156.

Abstract - In the experimental setting where pain or discomfort is elicited, it is imperative that the different types of stimuli used (i.e., chemical, mechanical, thermal, and electrical) be quantified and other important factors be considered: (1) reproducibility of the stimulus, which depends upon its ability to be completely characterized and exactly measured; and (2) control of potential modifying factors which might influence the pain threshold.

Thus, the stimulus must be measurable, reproducible, and behaviorally predictable.

\section{Major M. Ash}

University of Michigan School of Dentistry, Department of Occlusion, Ann Arbor, MI, USA

Key words: dentinal hypersensitivity, test stimuli, pain, pain induction.

Major M. Ash, D.D.S., University of Michigan School of Dentistry, Dept. of Occlusion, Ann Arbor, MI 48109-1078, U.S.A.

Accepted for publication 26 March 1986.
In order to properly assess degrees of clinical pain or discomfort, it is imperative that the stimulus be quantified. There exist a number of different types of stimuli which have been utilized in the experimental measurement of pain (1). They can be placed into such broad categories as mechanical, thermal, chemical and electrical stimuli, but vary in capacity to mimic the sensory, affective, or evaluative properties of a particular clinical disorder, including hypersensitivity of the teeth. A method of pain induction cannot be considered in isolation, inasmuch as interactions between the source of pain and pain attenuation may occur (2). From the same comparative perspective, quantification of stimuli should not be considered in isolation from variations in locus, energy, and method of determining thresholds.

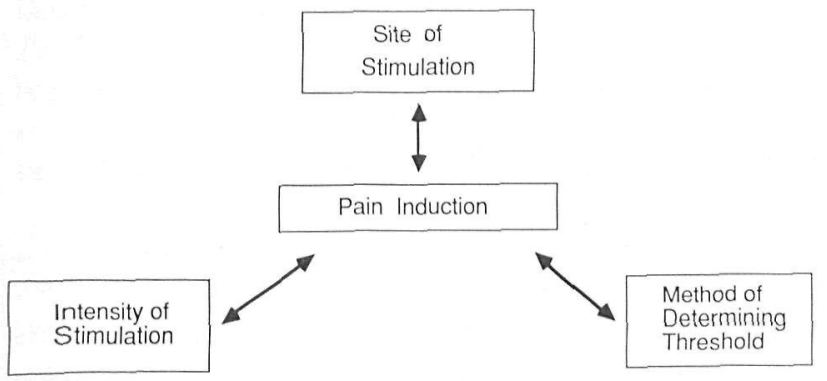

Because of the strong interactive effect of stimulus and subjective response, all reasonable control of external factors should be attempted. From this viewpoint, steps in quantifying various dental threshold stressors for testing the effects of treatment on dentinal hypersensitivity (3-7) do not appear to match the steps made in quantifying the multidimensional nature of nociception $(8,9)$.

Simple numerical category scales, which treat pain (discomfort, hypersensitivity) as a unitary phenomenon measured only in terms of magnitude, are perhaps adequate for assessing the amount of pain that is present, or relief of pain, but they are not sufficient to answer a variety of questions about pain intensity, its unpleasantness and its qualities of pressure, temperature, and vibration (10). However, even the use of simple scales for assessing pain requires that the inducing stimulus be measurable and reproducible and that the measurements be expressed in physical terms (amperes, millimeters, degrees centigrade, etc.)

$$
\begin{aligned}
& \text { Stimulus must be: } \\
& \text { - Measurable } \\
& \text { - Reproducible } \\
& \text { - Behavior Predictable }
\end{aligned}
$$

(Without quantification of the stimulus it is difficult, if not impossible, to compare the findings of different investigators). As already suggested, a stimulus must be reproducible and behave in a predictable or constant physical, chemical, thermal or electrical way during the period of stimulation. However, extraneous effects such as nonlinear coupling (viz. resistance, deformation, etc.) between the stimulus and sensory apparatus may influence the reproducibility of the threshold of pain. For example, the results of a recent laboratory study (11) on electrode placement suggest that the middle third of the incisors and the occlusal third of the premolars are the best electrode positions to stimulate the pulp at the lowest possible voltage without stimulation of nonpulpal fibers. However, these areas do not correspond physically with those of cervical hypersensitivity.

\section{Chemical stimuli}

Chemical stimuli have not been widely used because of inconvenience, difficulty of varying the stimulus 
intensity, potential injury to adjacent soft tissues, and the exclusion of substances which could cause injury to the hard tissues. There are no studies of dental cervical hypersensitivity in which the pain threshold was objectively determined by chemical stimuli.

\section{Mechanical (tactile) stimulus}

Only a few studies have used a "quantified" mechanical stimulus $(3,11,13)$ and the exact position of the sensitivity relocated with an occlusal key. An electronic pressure-controlled probe device (Yeaple Probe) modified from a periodontal probe (14) by replacing the tip with a dental \#16 explorer tip has been used. When a preset probing force is reached, the electromagnet holding the tip releases. The force applied begins with 10 grams and may be increased in $5 \mathrm{~g}$ increments until the first presence of discomfort, or $70 \mathrm{~g}$, is reached. The probing system can be calibrated. The intensity of the mechanical stimulus does not increase gradually, but rather in a saw-tooth form, with the force dropping to zero between incremental increases in mechanical force. "Scratching" of the surface may remove some of the desensitizing agent deposited on the surface of the tooth, but this has been reported not to substantially influence the pain threshold $(3,12)$. Analysis of the data from the Yeaple Probe requires an assumption that responses to greater than $70 \mathrm{~g}$ do not exist, or that no response is automatic to 70 g. This problem tends to defeat the use of a scaled stimulus.

\section{Thermal stimuli}

Quantified thermal (heat and cold) stimuli have been used $(3,12,13)$, as well as a cold air blast (7) $\left(1 \mathrm{~s}, 60 \mathrm{psi}, 70 \pm 3^{\circ} \mathrm{F}\right.$ ) to determine sensitivity levels before and after treatment for dentinal hypersensitivity. Variations in the distance from the tooth would influence the energy transfer involved in the cold air blast stimulus.

With the thermocouple device and cold air blast (7) the energy coupling between the delivery system and tooth suggests some unknown differences in the total amount of energy reaching the receptive field. Isolation and shielding of individual teeth and areas of teeth give some assurance that the actual amount of heat (or loss of it) has a linear relationship to the intensity of the stimulus.

The thermocouple device provides for a continuous spectrum of heat or cold to be applied at a point on the tooth via the tip of a probe containing a thermister which registers the temperature of the probe, but not (directly) the surface of the tooth. There is a lag between probe temperature and tooth surface temperature and this requires that changes in temperature be made slowly so the onset of sensitivity will not be bypassed. The device has sufficient range to apply cold (below $0^{\circ} \mathrm{C}$ ) and heat (above $50^{\circ} \mathrm{C}$ ) and therefore can test for sensitivity levels above and below initial baseline levels. In the complete absence of hypersensitivity following treatment, initial or normalized threshold values must be used.

The cold air blast (7) stimulus has quantification aspects of duration, known air temperature at the air jet, and known pressure (psi) at the nozzle of the air jet. How significant the differences that angulation of the jet, different distances from the tooth, or failure to "hit" a precise area are is not clear. In the absence of a stimulus of graded intensity, a change in the threshold of pain using physical values cannot be determined. It is possible to tell something about relief of pain provided that the stimulus is capable of inducing pain, which is not always the case. The degree to which thermal stimulation may also be considered mechanical in nature has not yet been answered.

\section{Electrical Stimulus}

An electrical stimulus has been used for some time to evaluate the "vitality" of the dental pulp. Problems in electrical pulp testing and dental algesimetry have been considered in a large number of publications and reviews may be found elsewhere (15-18). More recently, a device called the dentalpulp stethoscope was developed (4) which provides for digital RMS-G voltage stimulation. Thus, when a tingling sensation or warmth is first felt, the patient depresses a button to stop the stimulus and activate a recorder which prints the voltage level. A conductive gel with a $\mathrm{pH}$ of 5.4 to 5.6 is used. A value of 15 volts and above indicates a range of tooth nonsensitivity (19). There exists some controversy regarding the proper methodology to determine the pain threshold.

Pain threshold can be defined as the lowest electrical current (milliamperes) which, at a fixed frequency and impulse duration, first evokes a sensation (such as pricking) of pain (20). Stimulation of the pulp on the basis of applied voltage may not represent the exact pain threshold inasmuch as the stimulating current on an individual basis depends on varying resistance pathways to the pulp or to other adjacent tissues. By using a constant current source, the intensity of the stimulating current is independent of variable resistance paths (21). A procedure could be to slowly raise the stimulus current until the pain is first perceived, then give a series of 20 impulses at a time and measure the threshold again while the current is raised each time 
by $0.05 \mathrm{ma}$. Each recorded threshold would be the average of 3 or more measurements. As a control, "false impulses" could be given with the current held at zero increase. A pain stimulus need not "hurt" the patient. Reliable threshold sensations are dependent upon frequency and pulse duration. Standardized instructions are necessary.

Some concern exists within the research community relating to the use of a form of stimulus which is most unlike that associated with clinical pain. The potential for electrical shock or such a threat may influence the subject's assessment. With fear, there is an inclination toward a lower threshold. Distraction causes an increase in the pain threshold. Furthermore, although pain thresholds have been reported to vary no more than $15-20 \%$ between individuals when measured on skin (21), the degree of subject variation on the tooth surface is unclear.

\section{Reproducibility of the stimulus}

A stimulus that can be completely characterized and exactly measured is usually considered to be reproducible. As already discussed, the energy reaching the receptive field must be consistent with the energy of the applied stimulus. Nonlinear relationships, which cause variations in the transfer of energy, prevent any exact measurement of the intensity of the stimulus and therefore cannot be reliably reproduced. Calibration of a device in relation to physical measurements (degrees, grams, psi, etc.) provides assurance of a standard of comparison. However, such a procedure does not provide assurance that stimulus energy, for example voltage level, measured at the generating device, provides a constant current at the receptor field. The possibility that factors such as training, fatigue, temperature, distractions, fear, expectation, and procedural uncertainty may modify the pain threshold is a subject of considerable rescarch and controversy.

\section{Control of potential modifying influences}

A review of the potential factors which could possibly influence the pain threshold may be found elsewhere $(3,12,21)$. Some of the generally accepted precautionary measures to control extrancous influences include:

(1) Giving standardized instructions and demonstration of stimulus prior to baseline.

(2) The room should not be distracting because of noise, music, lights or temperature.

(3) Procedures which generate fear should be avoided.

(4) The patient should be allowed to come to "equi- librium" with the environment (a subject coming from a cold outside temperature to an inside temperature requires at least 15-20 min for the teeth to adjust before attempting to test sensitivity).

(5) Medications, mouthwashes, "cough drops", aspirin, etc. may influence the test.

(6) The use of appliances such as mouthguards and splints must be consistent with the time of the testing.

(7) Procedural testing, questioning, and instructions should be given prior to the time that baseline thresholds are determined.

\section{Application of stimulus}

Two aspects of applying the stimulus are considered here:

(1) Mode of applying a stimulus which can be varied in intensity; and

(2) The sequence of applying two or more kinds of stimuli.

The question of whether to apply the stimulus at random, or using a monotonic increase/decrease relates to the aspect of toleration and change in the pain threshold brought about by a number of above-threshold energy stimuli that would be delivered in a random order approach. The increase or decrease in the level of heat or increase in the level of electrical energy should be monotonic, although a continuous increase may not be possible. However, increments as well as continuous increases or decreases in stimulus strength should occur within a standard time frame.

The application of more than one kind of stimulus requires that the first does not detract from the second, nor the second from the third, etc. Cold stimulus should follow heat, the least disturbing stimulus first, and the stimulus which least parallels the patient's clinical complaint applied last. Subjects who do not have a complaint about a particular kind of irritant historically may respond to a particular stimulus at baseline measurement, but not again. Such stimuli should be positioned last. The order of stimulation should be determined at screening, prior to baseline measurements.

\section{Summary}

Quantification of the stimuli used for testing thresholds of pain (dentinal, cervical hypersensitivity) is an appropriate requirement for objective measurement of the effectiveness of treatment. Reproducibility of the stimulus requires that a linear or predictable relationship be demonstrated between the energy of the applied stimulus and the receptor field. 
Threshold does not necessarily mean the same thing for different stressors (stimuli), even though correlations tend to be statistically significant within a given pain source and across stressors. Objective measurement of thresholds requires quantification of the stimulus and informed judgement regarding the capacity of the stimulus to mimic the sensory, affective or evaluative aspects of dentinal hypersensitivity. The number of different effects that a form of energy (such as an air blast or electrical stimulus) can produce have not been clearly demonstrated. The selection of a stimulus should be based upon its being quantified, whether it subjectively needs to be most like clinical pain (dentinal hypersensitivity) or to provide the closest parallel to the affective and evaluative components of cervical hypersensitivity, and lastly upon what device is readily available. A number of factors which may alter the pain threshold should be controlled; most relate to procedural and environmental (clinic, laboratory) conditions which may distract the patient. Application of the stimulus should be by monotonic increase or decrease where the strength of the stimulus is variable. The sequence of applying two or more stimuli generally dictates that the least stressing and potentially least painful stimulus should be given first, or the stimulus with the least "carry over" effect should be given first. The final order should be determined prior to the time when baseline records are made.

\section{References}

1. Progacci P, Zoppi M, Maresca J. Experimental pain in man. Pain 1979; 6: 123.

2. Rollman GB. Measurement of experimental pain in chronic pain patients: Methodological and individual factors. In: Melzack R, ed. Pain measurement and assessment. New York: Raven Press, 1983.
3. Sмттн BA, Asn MM. A study of desensitizing dentrifices and cervical hypersensitivity. I Periodontol 1964; 35: 222.

4. Sтакк MM. Rationalization of electric pulp-testing mcthods. Oral Surg 1977; 4.3: 598.

5. Gangarosa LP, Park NH. Practical considerations in iontophoresis of fluoride for desensitizing dentin. I Prosthet Dent 1978; 39: 173

6. Jensen AL. Hypersensitivity controlled by iontophoresis: double-blind clinical investigation. JADA 1964; 68: 216.

7. Tarbet WJ. An evaluation of two methods for the quantitation of dentinal hypersensitivity. JADA 1979; 98: 914.

8. Gracely RH. Pain measurement in man. In: NG LKY, Bonica JJ, eds. Pain discomforl and humanitarian care. New York: Elsevier/North-Holland, 1980.

9. Heft MW, Parker SR. Perceptual scales for electrical tooth-pulp stimulation. I Dent Res $1981 ; 60(A): 383$.

10. Gracely RH. Pain language and ideal pain assessment. In: Melzask R, ed. Pain measurement and assessment. New York: Raven Press, 1983.

11. Jacobson JJ. Probe placement during electric pulp-testing procedures. Oral Surg 1984; 58: 242.

12. Sмгтн BA, Asн MM. Evaluation of a desensitizing dentrifice. JADA 1964; 68: 639.

13. Green BL. Calcium hydroxide and potassium nitrate as desensitizing agents for hypersensitive root surfaces. J Periodont 1977; 48: 667.

14. Caton J. Depth of periodontal probe penetration related to clinical and histologic signs of gingival inflammation. $f$ Periodontol 1981; 52: 626.

15. Mumfjord JM, Björn H. Problems in electric pulp testing and dental algesimetry. Int Dent J 1962; 12: 161.

16. Matrnews B. Thresholds of vital and nonvital teeth to stimulation with electric pulp testers. $\mathrm{Br}$ Dent f 1974; 137: 352.

17. BJörn H. Electrical excitation of teeth and its application to dentistry. Swed Dent J 1946; Suppl. 39.

18. Mumfjord JM. Pain perception, threshold, and adaptation of normal human teeth. Arch Oral Biol 1965; 10: 957.

19. Stark MM, Pelzner R. Measurement of dentinal hypersensitivity. Comp Cont Educ Dent 1982; S105, Suppl. 3.

20. Bonica JJ. The management of pain. Philadelphia: Lea and Febiger, 1953.

21. Notermans SLH. Measurements of the pain threshold determined by electrical stimulation and its clinical application. In: Weisenberg M, ed. Pain, clinical and experimental perspectives. St. Louis: C. V. Mosby Co., 1975. 
This document is a scanned copy of a printed document. No warranty is given about the accuracy of the copy. Users should refer to the original published version of the material. 